\title{
On the Color Stability of Phosphor-Converted White LEDs Under DC, PWM, and Bilevel Drive
}

\author{
K. H. Loo, Member, IEEE, Y. M. Lai, Member, IEEE, Siew-Chong Tan, Member, IEEE, \\ and Chi K. Tse, Fellow, IEEE
}

\begin{abstract}
Most commercial white LEDs are made from nitridebased blue LEDs coated with yttrium aluminium garnet phosphor, which produce spectra that shift in opposite directions under the influences of drive current and junction temperature changes. This property gives rise to different emitted spectra, hence chromaticity properties, when the LED is driven/dimmed by different current waveforms. By using a commercial white LED sample, LUXEON $\mathrm{K} 2$, the effects of drive current and junction temperature on the changes of chromaticity coordinates are studied experimentally. The impact of dc, pulse width modulation (PWM), and bilevel current waveform is discussed through a graphical analysis, followed by experimental verification. It is proven that dc offers the best color stability over dimming due to the counteracting influences of drive current and junction temperature variations, whereas an LED constantly suffers from noneliminable chromaticity changes when driven by the PWM. Theoretical explanations are given to justify these cases, and it is found that, for the case of dc drive, an ideal heat sink's thermal resistance can be selected based on a simple equation to minimize the overall chromaticity change over dimming. This paper provides an in-depth discussion on the relations between the chromaticity properties of phosphor-converted (pc) white LEDs and the driving/dimming methods used.
\end{abstract}

Index Terms-Driving method, LED, LED color, phosphorconverted (pc) LED, white LED.

\section{INTRODUCTION}

$\mathbf{N}$ ITRIDE-BASED blue LEDs emit a narrow-band emission at a peak wavelength of around $460 \mathrm{~nm}$ [1]. To yield a white light, part of the blue light is downconverted by phosphor to yellow light, and the two colors are mixed as they escape into the surrounding as a dichromatic white light. Since the blue light at $460 \mathrm{~nm}$ is well matched to the peak excitation spectrum of cerium (Ce)-doped yttrium aluminium garnets (YAGs) phosphor located at around $440-460 \mathrm{~nm}$, this method of producing white light offers good conversion efficiency and is used in nearly all commercial phosphor-converted (pc) white LEDs based on blue LED chips. However, for nitride-based blue LEDs, there are

Manuscript received May 13, 2010; revised August 24, 2010; accepted September 27, 2010. Date of current version January 9, 2012. This work was supported by the University Grants Committee of the Hong Kong Special Administrative Region, Research Grants Council Earmarked Research Grant under Grant PolyU 5272/07E. Recommended for publication by Associate Editor M. Ponce-Silva.

K. H. Loo is with the Faculty of Engineering, The Hong Kong Polytechnic University, Hong Kong (e-mail: kh.loo@polyu.edu.hk).

Y. M. Lai, S.-C. Tan, and C. K. Tse are with the Department of Electronic and Information Engineering, The Hong Kong Polytechnic University, Hong Kong (e-mail: enymlai@inet.polyu.edu.hk; ensctan@eie.polyu.edu.hk; encktse@inet.polyu.edu.hk).

Digital Object Identifier 10.1109/TPEL.2010.2086080 numerous experimental findings [2], [3] that reported the shift of peak emission wavelength with drive current and junction temperature. These effects unavoidably lead to color shift in the pc white LEDs. Under extreme variations in drive current and junction temperature, such as the case where the LEDs are operated over a wide dimming range, or when a poor thermal design is prescribed, the resulting color shift may exceed the acceptable limit used in the lighting industry and gives rise to eye-perceivable color change, thus leading to severe degradation in lighting quality.

Previous researchers have found that an increase in the drive current leads to a blueshift in peak emission wavelength (to shorter wavelengths) at constant junction temperature [2], [3]. This effect, in the case of III-V nitride-based LEDs, is due to the piezoelectricity-induced quantum confined Stark effect, as explained in [4], but some attribute it to the band-filling effect [3], [6]. On the other hand, an increase in the junction temperature at constant drive current leads to a redshift in peak emission wavelength (to longer wavelengths) due to the temperature dependence of semiconductor's bandgap energy [5]. Although the explanations to the origins of these phenomena are not unified, it is important to conclude from these experimental observations that drive current and junction temperature have counteracting effects on the peak wavelength shift of the blue light emitted by the nitride-based LEDs. Due to the wavelength-dependent absorption characteristic of phosphor, the mixing ratio between the absorbed and transmitted blue light changes under peak wavelength shift and leads to emitted light with different degrees of "white-ness." Therefore, in a practical dimmable lighting system, where changes in drive current and junction temperature occur frequently, a color shift in the pc white light is clearly present and should be remedied [7], [8].

To minimize the color shift in a pc white LED, an increase/decrease in the drive current leads to a certain degree of blueshift in peak emission wavelength that must be precisely compensated by the same degree of redshift induced by a corresponding increase/decrease in the junction temperature so that the overall wavelength shift is minimized. However, in a typical installation of white LED drive system, the drive current and junction temperature of LED are not two independently controllable parameters. With a fixed thermal design, different drive currents will cause different heating effects on the LED, which will naturally alter the junction temperature according to the heat flow balance, i.e., the rate of heat generation equals the rate of heat removal. Therefore, an exact red-/blueshift compensation is only possible if a suitable thermal design is implemented in order to achieve a color-stable pc white LED system. 
In this paper, we embark on an experimental study on the color shift of a commercial white LED as functions of drive current and junction temperature variations. The effects of each parameter on the LED color were measured by keeping the other parameter unchanged. These results provide the basis for discussion on the color shift properties of pc white LEDs when both parameters are varied simultaneously, a situation that is typically encountered in practical lighting systems. Using the conclusions from these results, we discuss the color shift scenarios when the LED is driven by dc, pulse width modulation (PWM) current, and bilevel (BL) current [9], [10]. The three driving methods are found to have different influences on the color shift properties of pc white LEDs, which pose design issues that should be considered by power electronics engineers for color-stable LED lighting systems. We also provide qualitative discussions to explain these effects, followed by experimental verification using a commercial white LED. Finally, a thermal design procedure based on heat sink's thermal resistance calculation is proposed for minimizing the color shift under each driving method.

\section{INFLUENCES OF DRIVE CURRENT AND JUNCTION TEMPERATURE}

In this section, an experiment was conducted to observe the color shift properties of a commercial white LED, LUXEON K2, under the influences of dc drive current and junction temperature changes. The LUXEON K2 produced by Philips-Lumileds is a typical phosphor-coated white LED based on indium-galliumnitride (InGaN) blue LED chip [11], which is known to suffer from peak emission wavelength shifts under different drive current and junction temperature conditions, and therefore is chosen as a representative sample of nitride-based LEDs for this experiment. (Note: The same experiment was conducted on another white LED sample, XLAMP XREWHT-L1, produced by CREE and similar observations were found; therefore, the results will not be discussed here to avoid duplication.) Due to the selective absorption characteristic of phosphor, longer wavelengths (due to redshift) are more readily absorbed by phosphor compared to shorter wavelengths (due to blueshift) [7]. This leads to chromaticity changes of the resulting pc white light under various operating conditions, which are reflected directly by the changes of chromaticity coordinates $(x, y, z)$. Hence, in this experiment, chromaticity coordinates are measured and their variations are used as indirect measurements of the red-/blueshift effects. Besides, since chromaticity coordinates are used to specify a color point on the International Commission on Illumination (CIE) chromaticity diagram, they give the most direct measurement of the color deviation of a test point from a reference.

The measurements were conducted using the experimental setup described in the following. To isolate the effects of drive current and junction temperature, the LUXEON K2 white LED sample is mounted on a temperature-controllable heat sink so that the junction temperature can be controlled independently. The heat sink's temperature $T_{\mathrm{hs}}$ required to generate a desired junction temperature $T_{j}$ for a given drive current $I_{f}$ can be estimated using the thermal resistance model described by (1), where $k_{h}$ is the portion of input electrical power $V_{f} I_{f}$ that is dis- sipated as heat. According to this model, for each drive current condition, the heat sink's temperature was adjusted to a value estimated from the LED's junction-to-case temperature drop, which, in turn, is determined from the product of the known junction-to-case thermal resistance $R_{j-c}$ (from datasheet) and the measured forward voltage $V_{f}$ and current $I_{f}$. In general, $k_{h}$ is not constant but varies nonlinearly with the power level of LED [12]. For LUXEON K2, the measurements in [12] showed that $k_{h}$ varies gradually from 0.85 to 0.90 for $67 \%$ to $100 \%$ of the rated power. Although experimental data are not available for $<67 \%$ of the rated power, the variation in $k_{h}$ is expected to be smaller in degree considering that the efficacy "droop" effect is not predominant at small forward currents and the luminous efficacy is approximately constant. Therefore, for the convenience of computation, a constant value of $k_{h}=0.85$ is used in this study, but the results computed based on this assumption and the theory proposed in Section IV should be subject to fine tuning when implemented in practice. Since thermal paste is used between the LED's case and the heat sink, $R_{c-\mathrm{hs}}$ is assumed to be small compared to the junction-to-case thermal resistance $R_{j-c}$ of the LED, $9\left[{ }^{\circ} \mathrm{C} / \mathrm{W}\right]$, specified in the datasheet [13].

$$
\begin{aligned}
T_{j} & =\left(R_{j-c}+R_{c-\mathrm{hs}}\right) k_{h} V_{f} I_{f}+T_{\mathrm{hs}} \\
& \approx R_{j-c} k_{h} V_{f} I_{f}+T_{\mathrm{hs}} .
\end{aligned}
$$

For optical measurements, the heat sink and LED assembly is housed inside a closed light chamber (to avoid interference from ambient light), where the emitted light from the LED is detected by a miniature fiber-optic spectrometer USB4000 produced by the Ocean Optics. The measured tristimulus values $X$ (red), $Y$ (green), and $Z$ (blue) are then used to calculate the chromaticity coordinates $(x, y, z)$ according to $x=X /(X+$ $Y+Z), y=Y /(X+Y+Z)$, and $z=1-x-y$, from which the chromaticity coordinates $x, y$, and $z$ can be viewed as the normalized degree of stimulation of the red, green, and blue cone of human eyes, respectively. In a dichromatic white light, since the degree of stimulation of the red and green cone, $x$ and $y$, both originate from the pc yellow light, they are expected to vary in phase with each other and, therefore, the following discussion can be based on two chromaticity coordinates $(x, z)$ only.

Fig. 1 shows the measured chromaticity coordinates $(x, y, z)$ as the dc forward current $I_{f}$ is varied from 100 to 1000 [mA] under three constant junction temperatures, i.e., 40, 60, and $80\left[{ }^{\circ} \mathrm{C}\right]$, where $x$ decreases and $z$ increases with increasing $I_{f}$. The reason for the observed trends is, as $I_{f}$ is increased, the blue light is emitted at shorter wavelengths (due to blueshift effect) and becomes less absorbed by the phosphor. This increases the amount of blue light that can be transmitted directly (by penetration through the phosphor) and enhances the blue component present in the detected white light. On the other hand, Fig. 2 shows that an opposite effect from that of drive current is induced by a variation in junction temperature $T_{j}$. This is caused by the redshift of the emitted blue light that alters the mixing ratio between the direct and phosphor emissions. At increasing $T_{j}$, red-shifted emission is more readily absorbed by phosphor and converted into yellow light, thus reducing the 


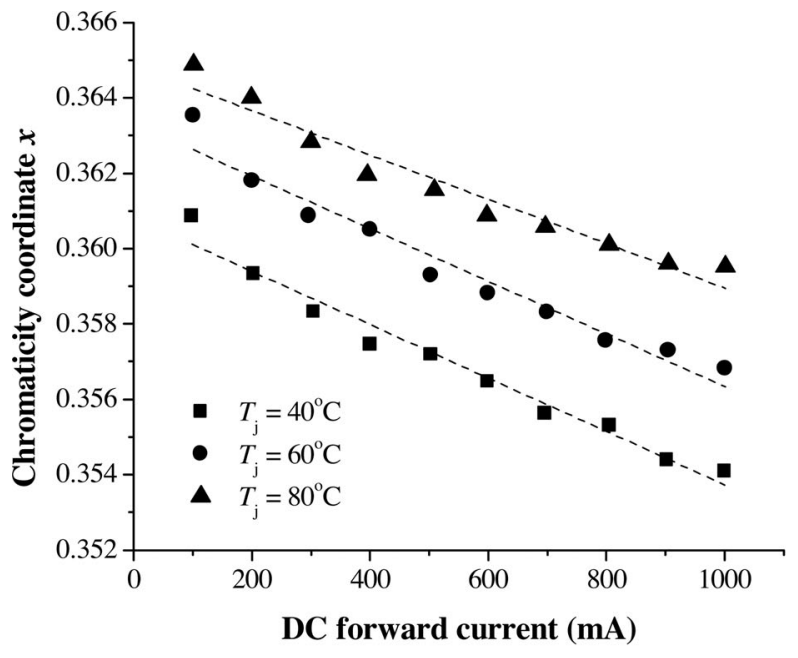

(a)

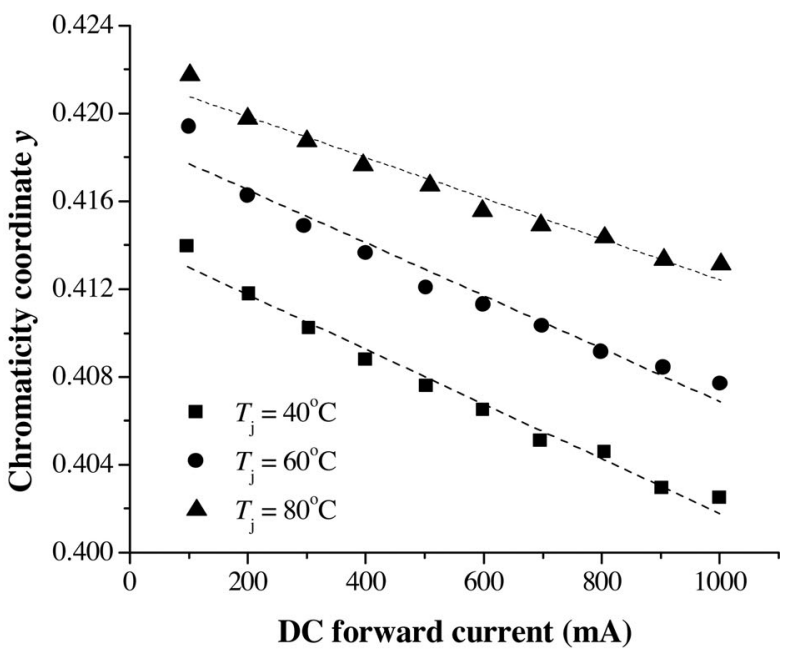

(b)

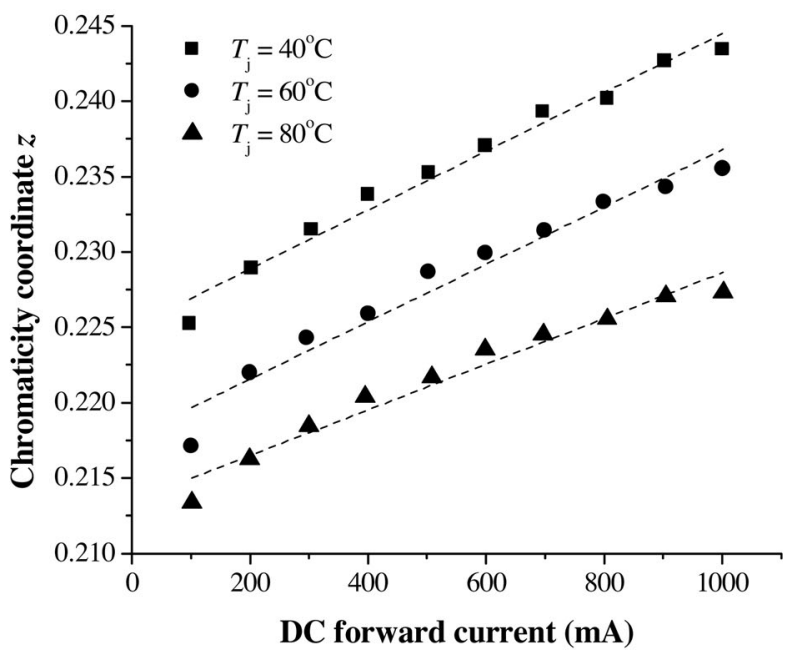

(c)

Fig. 1. Chromaticity coordinates [(a) $x$, (b) $y$, (c) $z$ ] measured on Philips-Lumileds LUXEON K2 (LXK2-PW14-U00, cool white) pc white LED sample under various dc forward currents. Dashed lines are linear fits to the experimental data.

amount of blue component present in the detected white light, as reflected by the decrease in $z$ and increase in $x$. The variations in chromaticity coordinates due to drive current, and junction temperature changes can also be represented by the shift of correlated color temperature (CCT) of the detected white light, as shown in Fig. 3. In general, a larger dc forward current or lower junction temperature tends to increase the CCT due to an enhanced blueshift effect that causes more blue light to be transmitted directly to the observers, giving the visual sensation of colder white light. Fig. 3(c) shows that the measured CCT varies linearly with the chromaticity coordinate $z$, hence the amount of blue component, and such a relationship is very weakly dependent on the junction temperature of LED. This agrees with the expectation that CCT reflects only the chromaticity property of the detected white light.

The information presented by these observations can be applied directly to any dimmable LED lighting system based on pc white LEDs. With a fixed thermal design, the junction temperature of a LED is determined by the heat conduction between the die and the ambient air through the LED's case and the heat sink. As the LED is driven up by increasing the drive current, the heat dissipated by the LED is increased and a higher junction temperature is established. Based on the observations made in the experiment earlier, one can deduce that a higher drive current causes a temporary blueshift in the peak emission, which is eventually "corrected" by the redshift induced by a higher junction temperature at the new steady state. Since the degree of redshift, and hence the rate of junction temperature rise, depends on the system's thermal design, it is possible to correctly balance the chromaticity changes due to drive current and junction temperature changes in a dimmable system by means of thermal design in order to obtain a color-stable white light. To achieve this objective, a suitable value of heat sink's thermal resistance $R_{\mathrm{hs}-a}$ should be chosen based on (2) and (3), where 


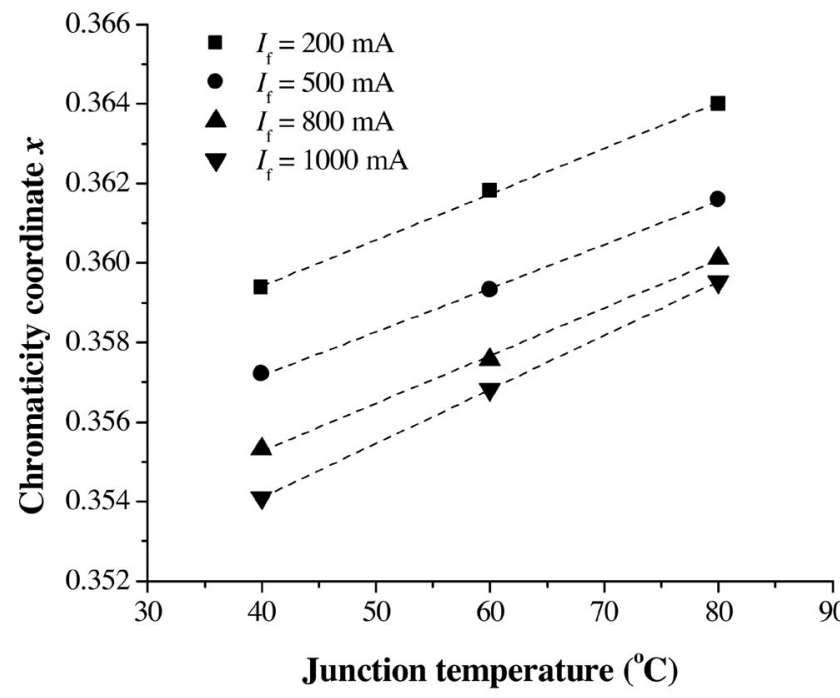

(a)

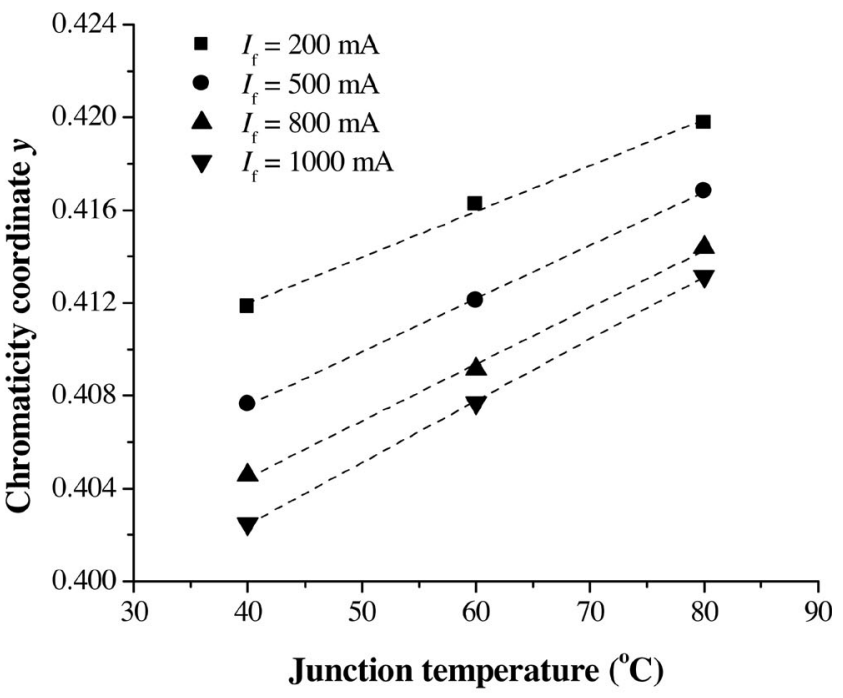

(b)

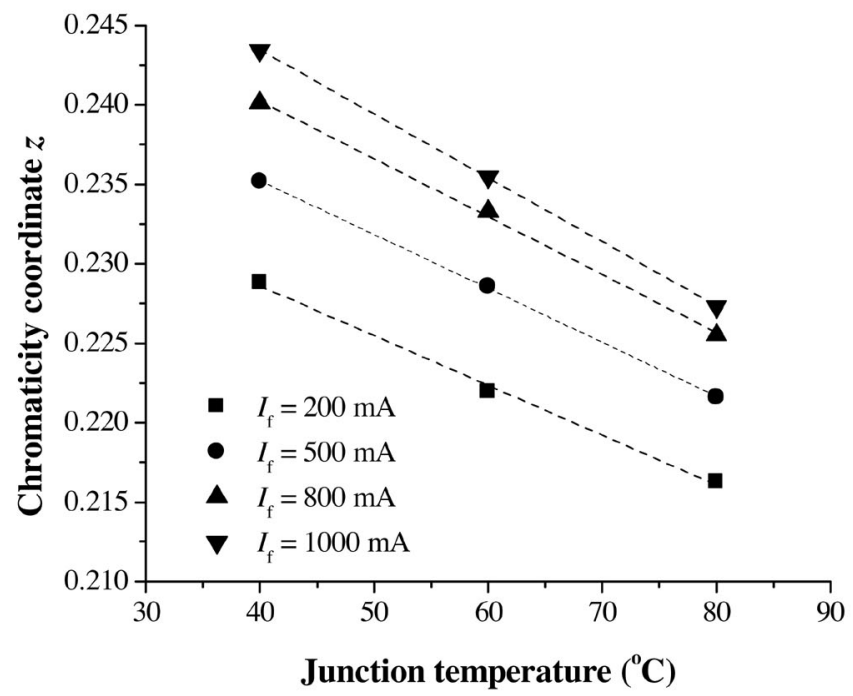

(c)

Fig. 2. Chromaticity coordinates [(a) $x$, (b) $y$, (c) $z]$ measured on Philips-Lumileds LUXEON K2 (LXK2-PW14-U00, cool white) pc white LED sample under various junction temperatures. Dashed lines are linear fits to the experimental data.

$T_{a}$ is the ambient temperature.

$$
\begin{aligned}
T_{j} & \approx\left(R_{j-c}+R_{\mathrm{hs}-a}\right) k_{\mathrm{h}} V_{f} I_{f}+T_{a} \\
\Delta T_{j} & \approx\left(R_{j-c}+R_{\mathrm{hs}-a}\right) k_{h} V_{f} \Delta I_{f} .
\end{aligned}
$$

\section{Chromaticity Changes Under Various Driving METHODS}

\section{A. Graphical Analysis}

It should be emphasized that the approach described by (2) and (3) is only valid for the dc-driven LED because the junction temperature is related directly to the current amplitude in such a case. When time-varying waveforms, such as PWM and BL current, are used, the junction temperature becomes a function of the average power (or current), in which case the current amplitudes (high and low) can be kept constant, while the average power (or current) is adjusted by varying the duty cycle.
Since the current amplitudes are fixed, the redshift caused by the variation in junction temperature is left uncompensated and the degree of chromaticity change depends only on the rate of junction temperature rise/fall with increasing/decreasing average power, and color shifts under such cases are always present and are impossible to be eliminated. The path of chromaticity change when the LED is driven by each current waveform is now described in greater depth.

Fig. 4 shows the path of chromaticity change when the LED is driven by varying dc current, in which case the average current has the same value as the current amplitude. In this scenario, as the LED is dimmed from the maximum current $I_{f, \max }, x$ will initially slide upward from $A$ to $A^{\prime}$ (step 1) since the thermal time constant is typically long and the junction temperature will momentarily remain constant at $T_{j, \max }$. However, since the LED power is decreased, the reduced heat dissipation will cause the junction temperature to fall slowly (step 2) and eventually settle 


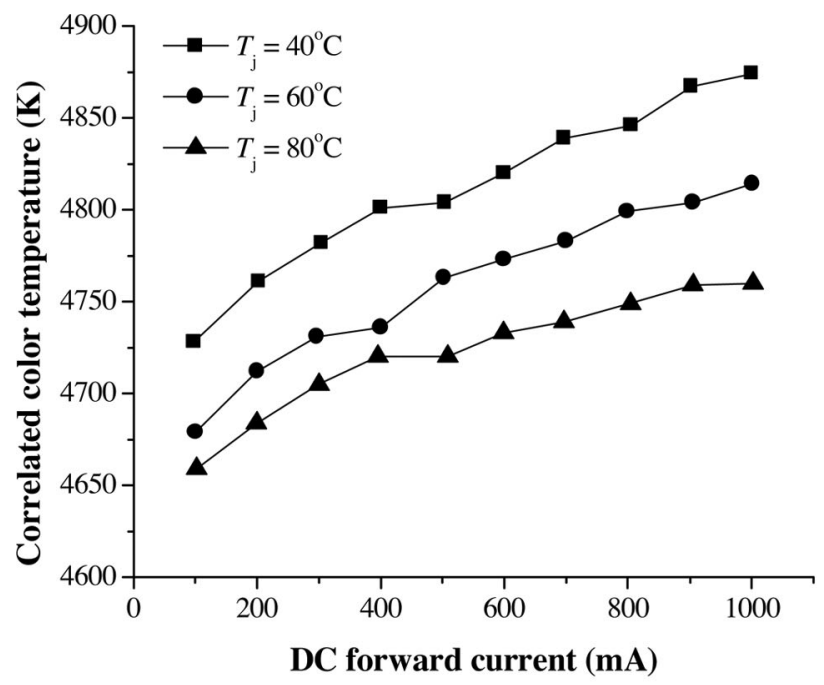

(a)

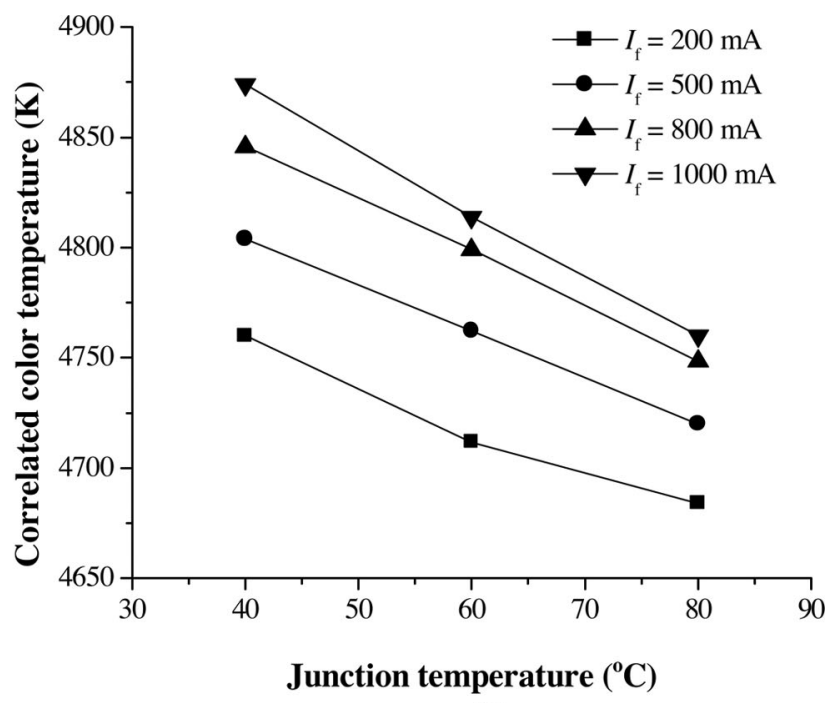

(b)

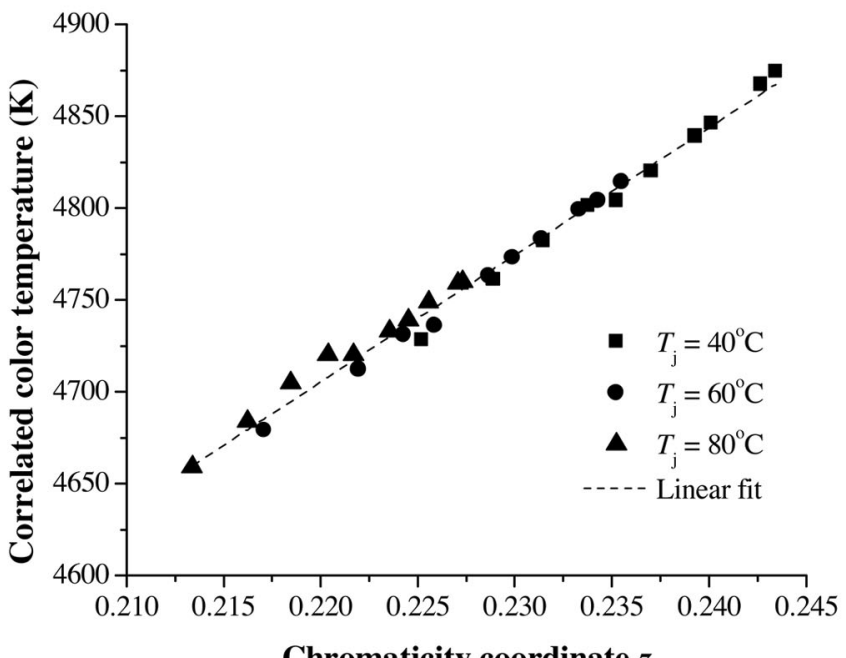

(c)

Fig. 3. CCT measured on Philips-Lumileds LUXEON K2 (LXK2-PW14-U00, cool white) pc white LED sample under various (a) dc forward currents and (b) junction temperatures. (c) Correlation between CCT and chromaticity coordinate $z$.

at a lower value at the new steady state denoted by $A^{\prime \prime}$. It can be deduced that if a suitable heat sink's thermal resistance is chosen, the change in $x$ that occurs during step 1 can be fully compensated by the same magnitude of negative change that occurs during step 2. This is equivalent to shifting the curves closer to each other until the line connecting $A$ and $A^{\prime \prime}$ becomes horizontal. The same concept applies to a full-range dimming from $A$ to $B$.

When the LED is driven by the PWM current, a very different path is traversed since the peak forward current $I_{f, \max }$ is fixed at all duty cycles $D$. As illustrated in Fig. 5, as the LED is dimmed by decreasing $D$ at constant $I_{f, \max }, x$ no longer slides upward but momentarily remains constant at $A$ until the reduced heat dissipation causes the junction temperature to fall slowly and eventually settle at $A^{\prime}$, where $x$ acquires a new steady-state value. In this case, $A^{\prime \prime}$ does not lie on the constant-temperature curve but is defined by the intersection point between the average current $\overline{I_{f}}\left(=D I_{f, \max }\right)$ and the value of $x$ at $A^{\prime}$. Under
PWM drive, once the value of $I_{f, \text { max }}$ is fixed, $x$ becomes a function of the junction temperature, and hence, the average power only. Therefore, any chromaticity change due to varying average current cannot be fully compensated by a selection of heat sink as is possible with the case of dc-driven LED, unless a constant junction temperature can be maintained by active temperature control method.

Fig. 6 shows the scenario when the LED is driven by BL current, which is the most complex case. Since BL current pulsates between two current levels, $I_{f, \max }$ and $I_{f, \min }$, varying the average current by adjusting the duty cycle $D$ gives rise to a linear mixing between the color sets contributed by each current level, which itself varies with the junction temperature as the average current is varied. Referring to Fig. 6, as the LED is dimmed from $A$ by decreasing the duty cycle $D$ from $D=1$, the junction temperature falls and the values of $x$ that correspond to $I_{f, \max }$ and $I_{f, \min }$ shift to $A^{\prime}$ and $B^{\prime}$, respectively, and the resultant $x$ (denoted by $A^{\prime \prime}$ ) is obtained from the duty-cycle-weighted 


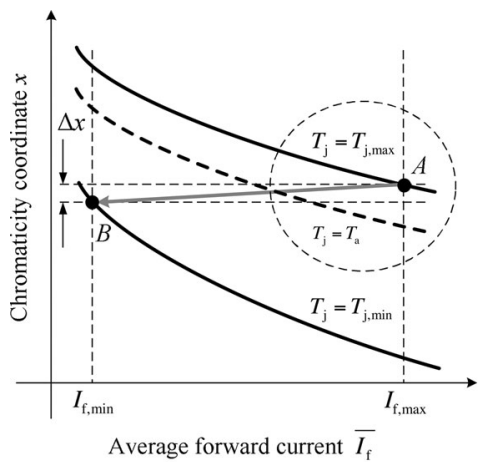

(a)

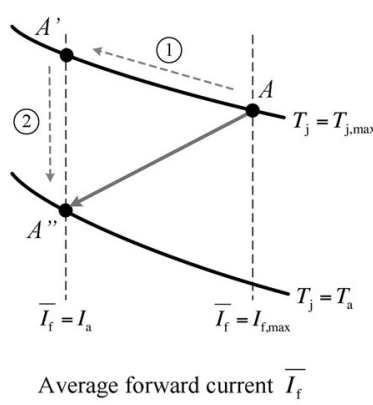

(b)
Fig. 4. Illustration of the path of variation of chromaticity coordinate $x$ under the simultaneous effects of dc drive current and junction temperature variations. (b) shows an enlarged view of the circled area in (a).

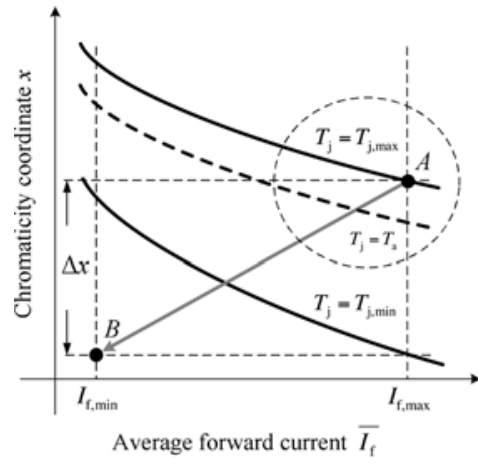

(a)

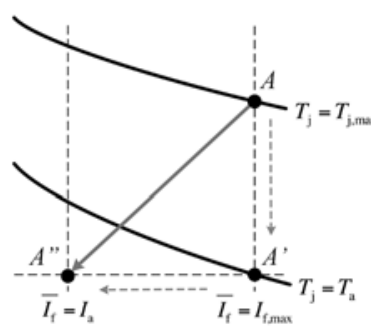

Average forward current $\overline{I_{\mathrm{f}}}$

(b)
Fig. 5. Illustration of the path of variation of chromaticity coordinate $x$ under the simultaneous effects of PWM drive current and junction temperature variations. (b) Shows an enlarged view of the circled area in (a).

average of $x\left(A^{\prime}\right)$ and $x\left(B^{\prime}\right)$, i.e., $x=D x\left(A^{\prime}\right)+(1-D) x\left(B^{\prime}\right)$. As $D$ continues to decrease, the change in junction temperature $\Delta T_{j} \approx\left(R_{j-c}+R_{\mathrm{hs}-a}\right) k_{h} V_{f} \Delta \overline{I_{f}}$ becomes increasingly smaller in magnitude compared to the ambient temperature $T_{a}$. Hence, the junction temperature no longer varies significantly with a further decrease in $D$ and the resultant $x$ gradually merges with the constant-temperature curve corresponding to $T_{j}=T_{j, \text { min }}$, where it finally reaches $B$ at $D=0$ or $\overline{I_{f}}=I_{f, \min }$. Similar to the PWM case, since both $I_{f, \max }$ and $I_{f, \min }$ are fixed in levels, the chromaticity of a BL-driven LED changes only with the junction temperature and color mixing at various duty cycles, thus it is impossible to eliminate the color shift that occurs over the dimming range by simple thermal design only.

From the graphical analysis discussed earlier, one can deduce that as the average current of an LED is varied over the full dimming range with a fixed thermal design, the most stable white light is obtained when the LED is driven by dc current, whereas the worst color stability occurs when it is driven by PWM current. This is verified by the following experiment.

\section{B. Experimental Verification}

Fig. 7 shows the measured chromaticity coordinate $x$ when the white LED sample (LUXEON K2) is driven by the three

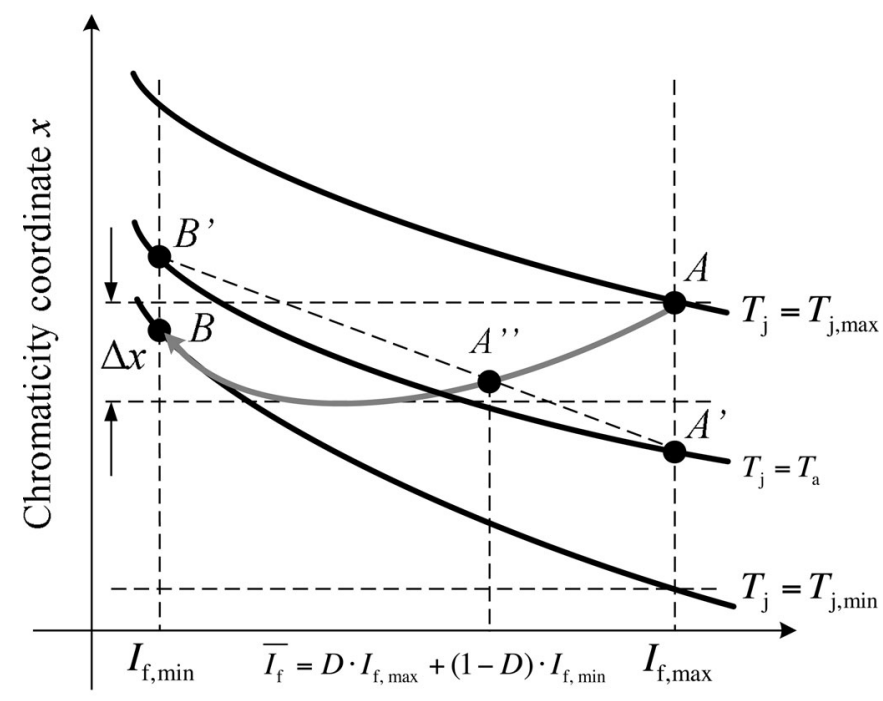

Average forward current $\overline{I_{\mathrm{f}}}$

Fig. 6. Illustration of the path of variation of chromaticity coordinate $x$ under the simultaneous effects of BL drive current and junction temperature variations.

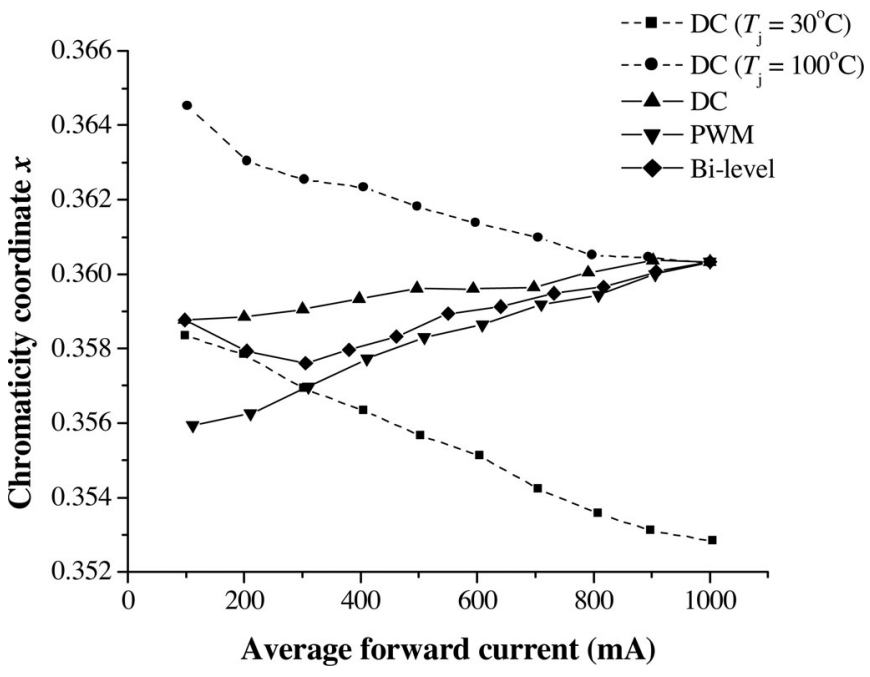

Fig. 7. Measured chromaticity coordinate $x$ when the white LED sample (LUXEON K2) is driven by three types of current waveforms (dc, PWM, and $\mathrm{BL}$ ). The measured data at two constant junction temperatures, $T_{j}=30^{\circ} \mathrm{C}$ and $T_{j}=100^{\circ} \mathrm{C}$, are included for references.

types of current waveforms discussed in Section III-A. The thermal resistance of the aluminium heat sink is kept constant at $13.4\left[{ }^{\circ} \mathrm{C} / \mathrm{W}\right]$ in all cases, except for the two sets of data measured at constant junction temperatures $T_{j}=30\left[{ }^{\circ} \mathrm{C}\right]$ and $T_{j}=100\left[{ }^{\circ} \mathrm{C}\right]$ where the heat sink's temperature is actively controlled for constant $T_{j}$. It is evident from the measured trends that the chromaticity coordinate $x$ has the most stable value over the full dimming range when the LED is driven by dc current. This is due to the counteracting effect between the peak emission wavelength shift induced by drive current and junction temperature variation, respectively, as explained earlier. This effect is not present in the case of PWM-driven LED since the 
current levels $\left(0, I_{f, \max }\right)$ are fixed in amplitudes, and therefore, $x$ varies monotonically with junction temperature only. It can be seen that, in the region close to the maximum average current $\overline{I_{f}}=I_{f, \max }$, both PWM- and BL-driven LEDs produce similar $x$ values due to the dominance of $I_{f, \max }$ at near-to-unity duty cycle. As the average current is decreased, the contribution from $I_{f \text {, min }}$ gradually increases and the value of $x$ produced by BL current starts to deviate from that produced by PWM current, until it finally merges with that produced by dc current at $I_{f, \min }$ when zero duty cycle is reached.

The graphical analysis and experimental results presented earlier point to a completely different view from the one commonly adopted by power electronics engineers [14]-[22], that PWM driving/dimming offers the best color stability due to the use of constant current levels, while the opposite is true for $\mathrm{dc}$ driving/dimming. The shortcoming of this traditional view is that the effect of junction temperature is clearly not taken into account or not carefully isolated from the effect of drive current [23], [24]. Although we focus on the pc white light produced by InGaN blue LEDs, Mukai et al. [3] found that the same spectral dependencies on drive current and junction temperature were observed on InGaN green and AlInGaP red LEDs. Therefore, the preceding discussion on the impact of different driving/dimming methods on color stability can also be applied to the production of white light from red, green, and blue (RGB) LEDs.

\section{Minimization of Chromaticity Change}

In this section, we proceed to identify the condition under which the chromaticity change is minimized for each driving/dimming method when the influences of drive current and junction temperature are present simultaneously. To conform to the CIE chromaticity diagram, the chromaticity coordinates $(x, y)$ are used in the discussion.

\section{A. General Formulation}

The chromaticity coordinates $(x, y)$ are functions of the drive current $I_{f}$ and junction temperature $T_{j}$ of an LED, $x=x\left(I_{f}, T_{j}\right)$ and $y=y\left(I_{f}, T_{j}\right)$. Hence, for a small change in $I_{f}$ and $T_{j}$, the resulting change in the individual chromaticity coordinate is given by

$$
\begin{aligned}
& d x=\frac{\partial x}{\partial I_{f}} d I_{f}+\frac{\partial x}{\partial T_{j}} d T_{j} \\
& d y=\frac{\partial y}{\partial I_{f}} d I_{f}+\frac{\partial y}{\partial T_{j}} d T_{j}
\end{aligned}
$$

The chromaticity change of a light source is more generally measured by the geometrical distance $\sqrt{\epsilon}$ between two color points on the chromaticity diagram, which includes both $d x$ and $d y$, as given by (6).

$$
\epsilon=(d x)^{2}+(d y)^{2}
$$

Substituting (4) and (5) into (6) gives

$$
\epsilon=\lambda_{1}\left(d I_{f}\right)^{2}+\lambda_{2}\left(d I_{f}\right)\left(d T_{j}\right)+\lambda_{3}\left(d T_{j}\right)^{2}
$$

where

$$
\begin{aligned}
& \lambda_{1}=\left(\frac{\partial x}{\partial I_{f}}\right)^{2}+\left(\frac{\partial y}{\partial I_{f}}\right)^{2} \\
& \lambda_{2}=2\left(\frac{\partial x}{\partial I_{f}} \frac{\partial x}{\partial T_{j}}+\frac{\partial y}{\partial I_{f}} \frac{\partial y}{\partial T_{j}}\right) \\
& \lambda_{3}=\left(\frac{\partial x}{\partial T_{j}}\right)^{2}+\left(\frac{\partial y}{\partial T_{j}}\right)^{2} .
\end{aligned}
$$

Since $x$ and $y$ measure the degree of red and green stimulations, respectively, which originate from the same phosphor emission, they are expected to vary in phase with each other. Hence, two proportionality constants $\alpha$ and $\beta$ can be introduced such that

$$
\begin{gathered}
\alpha=\frac{\partial y / \partial I_{f}}{\partial x / \partial I_{f}} \\
\beta=\frac{\partial y / \partial T_{j}}{\partial x / \partial T_{j}} .
\end{gathered}
$$

Substituting $\alpha$ and $\beta$ into (8)-(10) gives

$$
\begin{aligned}
& \lambda_{1}=\left(1+\alpha^{2}\right)\left(\frac{\partial x}{\partial I_{f}}\right)^{2} \\
& \lambda_{2}=2(1+\alpha \beta) \frac{\partial x}{\partial I_{f}} \frac{\partial x}{\partial T_{j}} \\
& \lambda_{3}=\left(1+\beta^{2}\right)\left(\frac{\partial x}{\partial T_{j}}\right)^{2} .
\end{aligned}
$$

1) Case 1: DC Drive: For an LED mounted on a heat sink with thermal resistance $R_{\mathrm{hs}-a}$, the junction temperature $T_{j}$ is related to the de drive current $I_{f}$ by (2). Using this simple thermal model, when $I_{f}$ is varied by a small magnitude $d I_{f}, T_{j}$ changes by $d T_{j}$ as given by (16). For an exact calculation, the dependence of $V_{f}$ on $I_{f}$ should be included, but this will give rise to a formidable task that cannot be solved analytically. Therefore, for the purpose of illustration of concept, $V_{f}$ is approximated by the nominal forward voltage $V_{f}=3.4$ [V] [13].

$$
\begin{aligned}
d T_{j} & =\left(R_{j-c}+R_{\mathrm{hs}-a}\right) k_{h} V_{f} d I_{f} \\
& =\Omega d I_{f}
\end{aligned}
$$

where $\Omega=\left(R_{j-c}+R_{\mathrm{hs}-a}\right) k_{\mathrm{h}} V_{f}$ is a constant. Substituting (16) into (7) gives

$$
\epsilon=\left(\lambda_{1}+\lambda_{2} \Omega+\lambda_{3} \Omega^{2}\right)\left(d I_{f}\right)^{2} .
$$

The value of $\Omega$ that gives a minimum $\epsilon$ is obtained from the first derivative of the quadratic equation in $\Omega$ provided $d I_{f} \neq 0$, which gives

$$
\Omega=\frac{d T_{j}}{d I_{f}}=-\frac{\lambda_{2}}{2 \lambda_{3}}=-\left(\frac{1+\alpha \beta}{1+\beta^{2}}\right)\left(\frac{\partial x / \partial I_{f}}{\partial x / \partial T_{j}}\right) .
$$

The corresponding minimum (since $\lambda_{3}>0$ ) chromaticity change $\epsilon$ is given by (19).

$$
\left.\epsilon\right|_{\min }=\frac{(\alpha-\beta)^{2}}{1+\beta^{2}}\left(\frac{\partial x}{\partial I_{f}}\right)^{2}\left(d I_{f}\right)^{2} .
$$


It can be easily shown that $\epsilon$ can be reduced to zero (absolute minimum) for the special case $\alpha=\beta$, under which the quadratic equation has equal roots at $\Omega=-\lambda_{2} / 2 \lambda_{3}$. This condition is met when the effects of drive current and junction temperature are equal in magnitude but opposite in direction. By using $\partial x / \partial I_{f}=-6.64 \times 10^{-3}\left[\mathrm{~A}^{-1}\right], \partial x / \partial T_{j}=$ $1.20 \times 10^{-4}\left[{ }^{\circ} \mathrm{C}^{-1}\right], \alpha=1.69$, and $\beta=1.96$ from the measured data shown in Figs. 1 and 2, and $R_{j-c}=9\left[{ }^{\circ} \mathrm{C} / \mathrm{W}\right]$, $k_{\mathrm{h}}=0.85$, and $V_{f}=3.4[\mathrm{~V}]$, the required heat sink's thermal resistance $R_{\mathrm{hs}-a}$ that gives a minimum $\epsilon$ is

$$
R_{\mathrm{hs}-a}=8.1\left[{ }^{\circ} \mathrm{C} / \mathrm{W}\right] .
$$

2) Case 2: PWM Drive: The case of PWM drive can be analyzed similarly by substituting $d I_{f}=0$ into (7) since the peak current level is fixed. With PWM current, the change in junction temperature $T_{j}$ is directly related to the change in average power, hence the change in duty cycle $D$. Thus,

$$
\begin{aligned}
d T_{j} & =\left(R_{j-c}+R_{\mathrm{hs}-a}\right) k_{h} V_{f} \cdot I_{f} d D \\
& =\Omega^{\prime} d D .
\end{aligned}
$$

Substituting $d I_{f}=0$ and (21) into (7) gives

$$
\epsilon=\lambda_{3}\left(\Omega^{\prime}\right)^{2}(d D)^{2} .
$$

Equation (22) shows that the chromaticity change $\epsilon$ can be minimized by: 1) keeping the duty cycle constant, i.e., $d D=0$, which is a trivial case, or 2) having a perfectly conducting thermal path from the LED to the ambient, i.e., $\left(R_{j-c}+R_{\mathrm{hs}-a}\right)=0$, which is an unrealistic case. When the thermal resistances are fixed, $\epsilon$ is directly proportional to $(d D)^{2}$. This proves that chromaticity change cannot be minimized when an LED is dimmed by the PWM.

3) Case 3: Bilevel Drive: When an LED is driven by a BL current that pulsates between $I_{H}$ (maximum) and $I_{L}$ (minimum), the resultants $x$ and $y$ is given by (23) and (24), respectively, where $D$ is the duty cycle applied to $I_{H}$.

$$
\begin{aligned}
& x=D x\left(I_{H}, T_{j}\right)+(1-D) x\left(I_{L}, T_{j}\right) \\
& y=D y\left(I_{H}, T_{j}\right)+(1-D) y\left(I_{L}, T_{j}\right) .
\end{aligned}
$$

Since $I_{H}$ and $I_{L}$ are fixed in levels, i.e., $d I_{H}=d I_{L}=0$, it can be shown that

$$
\begin{aligned}
& d x=\Delta x d D+\frac{\partial x}{\partial T_{j}} d T_{j} \\
& d y=\Delta y d D+\frac{\partial y}{\partial T_{j}} d T_{j}
\end{aligned}
$$

where $\Delta x=x\left(I_{H}, T_{j}\right)-x\left(I_{L}, T_{j}\right)$ and $\Delta y=y\left(I_{H}, T_{j}\right)-$ $y\left(I_{L}, T_{j}\right)$, both of which are approximately constant for all junction temperatures as can de deduced empirically from Figs. 1 and 2. Physically, this implies that the blue- and redshift effects have their origins at distinct physical phenomena, and therefore, the influences of drive current and junction temperature are mutually independent. Substituting (25) and (26) into (6) gives

$$
\epsilon=\lambda_{1}(d D)^{2}+\lambda_{2}(d D)\left(d T_{j}\right)+\lambda_{3}\left(d T_{j}\right)^{2}
$$

where

$$
\begin{aligned}
\alpha & =\frac{\Delta y}{\Delta x} \\
\beta & =\frac{\partial y / \partial T_{j}}{\partial x / \partial T_{j}} \\
\lambda_{1} & =\left(1+\alpha^{2}\right)(\Delta x)^{2} \\
\lambda_{2} & =2(1+\alpha \beta) \Delta x \frac{\partial x}{\partial T_{j}} \\
\lambda_{3} & =\left(1+\beta^{2}\right)\left(\frac{\partial x}{\partial T_{j}}\right)^{2} .
\end{aligned}
$$

Similar to the case of PWM, the change in junction temperature $T_{j}$ is directly related to the change in duty cycle $D$. Thus,

$$
\begin{aligned}
d T_{j} & =\left(R_{j-c}+R_{\mathrm{hs}-a}\right) k_{h} V_{f}\left(I_{H}-I_{L}\right) d D \\
& =\Omega^{\prime \prime} d D .
\end{aligned}
$$

Substituting (33) into (27) gives

$$
\epsilon=\left[\lambda_{1}+\lambda_{2}\left(\Omega^{\prime \prime}\right)+\lambda_{3}\left(\Omega^{\prime \prime}\right)^{2}\right](d D)^{2}
$$

which is similar to (17) for dc drive. Hence, the value of $\Omega^{\prime \prime}$ that gives a minimum $\epsilon$ is obtained from the first derivative of the quadratic equation in $\Omega^{\prime \prime}$, which gives

$$
\Omega^{\prime \prime}=-\frac{\lambda_{2}}{2 \lambda_{3}}=-\left(\frac{1+\alpha \beta}{1+\beta^{2}}\right)\left(\frac{\Delta x}{\partial x / \partial T_{j}}\right) .
$$

By using $\quad \Delta x=-6.00 \times 10^{-3}, \quad \partial x / \partial T_{j}=1.20 \times$ $10^{-4}\left[{ }^{\circ} \mathrm{C}^{-1}\right], \alpha=1.69$, and $\beta=1.96$ from the measured data shown in Figs. 1 and 2, and $R_{j-c}=9\left[{ }^{\circ} \mathrm{C} / \mathrm{W}\right], k_{\mathrm{h}}=0.85$, $V_{f}=3.4[\mathrm{~V}], \quad I_{H}=1000[\mathrm{~mA}], \quad$ and $\quad I_{L}=100[\mathrm{~mA}]$, the required heat sink's thermal resistance $R_{\mathrm{hs}-a}$ that gives a minimum $\epsilon$ is

$$
R_{\mathrm{hs}-a}=8.1\left[{ }^{\circ} \mathrm{C} / \mathrm{W}\right] .
$$

\section{B. Impact of Different Thermal Resistance Values}

To verify the validity of (18) for selecting the heat sink's thermal resistance that minimizes the chromaticity change under dc drive, $x, y$, and $\sqrt{\epsilon}$ were calculated for $R_{\mathrm{hs}-a}=8.1\left[{ }^{\circ} \mathrm{C} / \mathrm{W}\right]$ (from Section IV-A1). The case of dc drive is considered here for it provides the best color stability among the three driving/dimming methods discussed. The calculations were performed using the best-fitted lines, as shown in Fig. 1, and the thermal model described by (2). Fig. 8 shows the calculated data with the measured data included for comparisons. It can be seen that the calculated and measured chromaticity coordinates agree well with each other, implying that the thermal model is reasonably accurate for predicting the LED's junction temperatures under different drive currents. Although the change in chromaticity coordinates $x$ and $y$ is not individually minimized, it is evident that the overall chromaticity change $\epsilon$ is significantly reduced when the heat sink's thermal resistance is varied from 13.4 to $8.1\left[{ }^{\circ} \mathrm{C} / \mathrm{W}\right]$, and remains small over the 


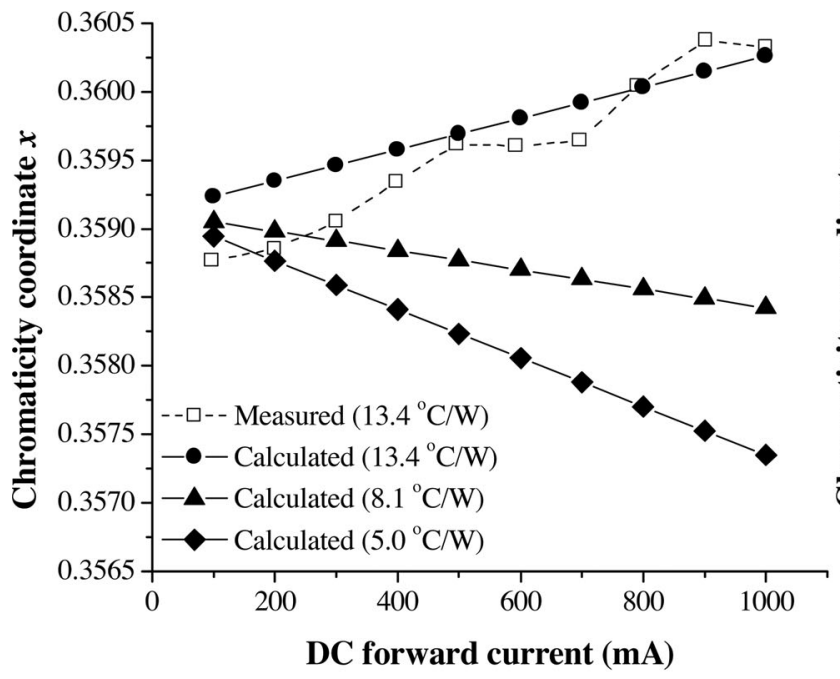

(a)

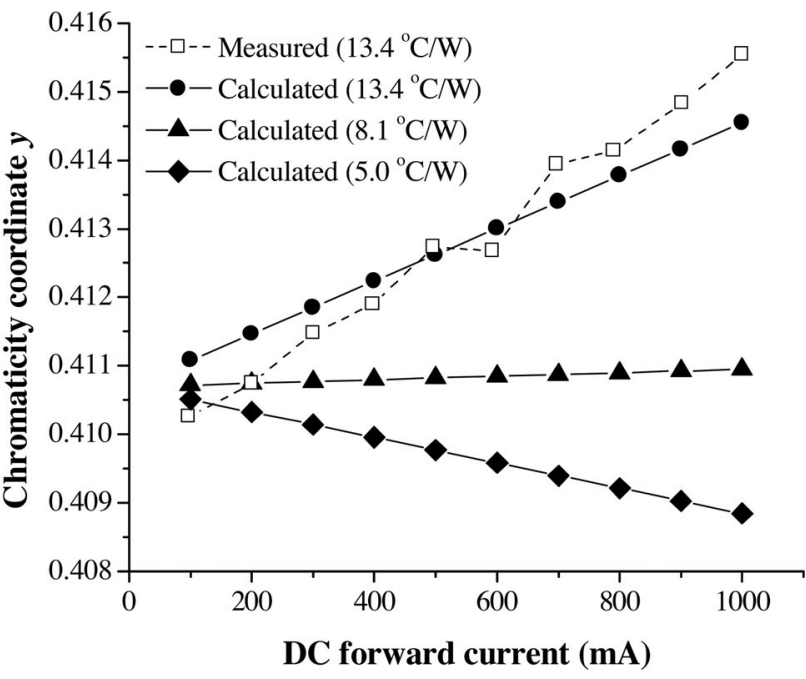

(b)

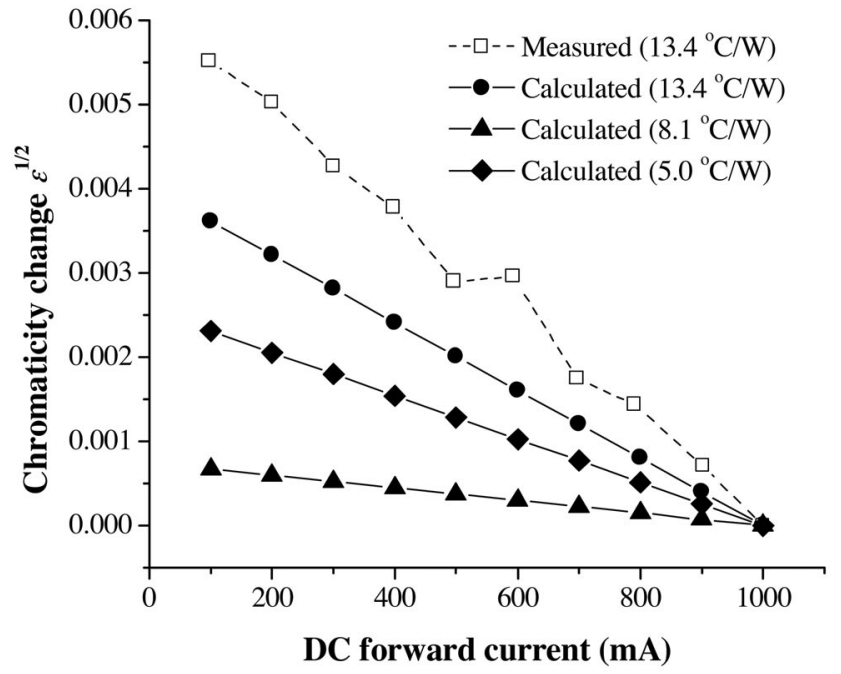

(c)

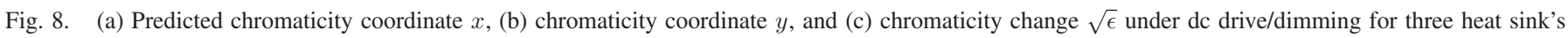
thermal resistance values.

full dimming range. It should be noted that smaller thermal resistance values do not necessarily lead to smaller chromaticity changes, for they will weaken the influence of junction temperature and cause insufficient compensation to the influence of drive current variation, as shown by the calculated data for $R_{\mathrm{hs}-a}=5.0\left[{ }^{\circ} \mathrm{C} / \mathrm{W}\right]$.

From Fig. 8, it can be seen that although the discrepancies between the measured and calculated data are relatively small, they are clearly not negligible. These effects are likely to arise from the deviation of $\partial(x, y) / \partial\left(I_{f}, T_{j}\right)$ from the assumed linearity, and the exclusion of nonlinear effects in the simplified thermal model, such as the variation of $k_{h}$ with LED power [12] and the complex dependencies of $R_{\mathrm{jc}}$ on LED power, LED size and mounting structure, heat sink's thermal resistance and orientation, and ambient temperature [25], which are not considered in the present theory to enable an analytically tractable problem to be formulated and an approximate solution to be found. Con- sequently, the proposed theory should produce with confidence a reasonable initial guess to the "optimum" heat sink's thermal resistance (that gives a minimum $\epsilon$ ), but users are required to fine tune this value to account for the discrepancies discussed earlier.

\section{CONCLUSION}

The effects of drive current and junction temperature variations on the chromaticity changes of nitride-based pc white LEDs were discussed. From the measured chromaticity data on a commercial white LED sample, it was proven that drive current and junction temperature variations have opposite effects on the changes of chromaticity coordinates. This property gives rise to different chromaticity shift scenarios when the LED is driven/dimmed by different current waveforms. The paths of chromaticity coordinate variations were discussed for the cases of dc, PWM, and BL current drive. The concepts were verified 
by experimental measurements, from which it was proven that $\mathrm{dc}$ and PWM offer the best and the worst color stability, respectively, over dimming, a conclusion that is in strong opposition to the generally adopted view. Theoretical formulations were developed to justify these conclusions, and, for the case of dc drive, the design procedure for selecting the heat sink's thermal resistance that minimizes the overall chromaticity change was presented. This procedure was shown to be accurate and can be conveniently adopted for improving the color stability in all pc white LED-based lighting systems.

\section{ACKNOWLEDGMENT}

The authors would like to thank W. K. Lun, K. T. Mok, and S. K. Ng for their assistance on the experimental setup and discussion.

\section{REFERENCES}

[1] M. R. Krames, O. B. Shchekin, R. Mueller-Mach, G. O. Mueller, L. Zhou, G. Harbers, and M. G. Craford, "Status and future of high-power lightemitting diodes for solid-state lighting," J. Display Technol., vol. 3, no. 2, pp. 160-175, Jun. 2007.

[2] S. Nakamura, "InGaN/AlGaN blue-light-emitting diodes," J. Vacuum Sci. Technol. A, vol. 13, no. 3, pp. 705-710, May/Jun. 1995.

[3] T. Mukai, M. Yamada, and S. Nakamura, "Current and temperature dependences of electroluminescence of InGaN-based UV/Blue/Green lightemitting diodes," Jpn. J. Appl. Phys. Part 2, vol. 37, no. 11B, pp. L1358L1361, Dec. 1998.

[4] T. Takeuchi, S. Sota, M. Katsuragawa, M. Komori, H. Takeuchi, H. Amano, and I. Akasaki, "Quantum-confined stark effect due to piezoelectric fields in GaInN strained quantum wells," Jpn. J. Appl. Phys. Part 2, vol. 36, no. 4A, pp. L382-L385, Apr. 1997.

[5] E. Fred Schubert, Light-Emitting Diodes. Cambridge, U.K.: Cambridge University Press, 2003.

[6] Y. Narukawa, I. Niki, K. Izuno, M. Yamada, Y. Murazaki, and T. Mukai, "Phosphor-conversion white light emitting diode using InGaN nearultraviolet chip," Jpn. J. Appl. Phys. Part 2, vol. 41, no. 4A, pp. L371L373, Apr. 2002.

[7] R. Mueller-Mach, G. O. Mueller, M. R. Krames, and T. Trottier, "Highpower phosphor-converted light-emitting diodes based on III-nitrides," IEEE J. Sel. Topics Quantum Electron., vol. 8, no. 2, pp. 339-345, Mar./Apr. 2002.

[8] H. Masui, S. Nakamura, and S. Denbaars, "Effects of phosphor application geometry on white light-emitting diodes," Jpn. J. Appl. Phys. Part 2, vol. 45, no. 34, pp. L910-L912, Sep. 2006.

[9] W. K. Lun, K. H. Loo, S. C. Tan, Y. M. Lai, and C. K. Tse, "Bilevel current driving technique for LEDs," IEEE Trans. Power Electron., vol. 24, no. 12, pp. 2920-2932, Dec. 2009.

[10] K. H. Loo, W. K. Lun, S. C. Tan, Y. M. Lai, and C. K. Tse, "On driving techniques for LEDs: Toward a generalized methodology," IEEE Trans. Power Electron., vol. 24, no. 12, pp. 2967-2976, Dec. 2009.

[11] Technology white paper, Understanding Power LED Lifetime Analysis, Philips Lumileds.

[12] S. Y. (Ron) Hui and Y. X. Qin, "A general photo-electro-thermal theory for light emitting diode (LED) systems," IEEE Trans. Power Electron., vol. 24, no. 8, pp. 1967-1976, Aug. 2009.

[13] Technical datasheet DS51, LUXEON K2, Philips Lumileds.

[14] J. Garcia, M. A. Dalla-Costa, J. Cardesin, J. M. Alonso, and M. RicoSecades, "Dimming of high-brightness LEDs by means of luminous flux thermal estimation," IEEE Trans. Power Electron., vol. 24, no. 4, pp. 1107-1114, Apr. 2009.

[15] B. Ackermann, V. Schulz, C. Martiny, A. Hilgers, and X. Zhu, "Control of LEDs," in Proc. Conf. Record 2006 IEEE Ind. Appl. Conf., 2006, 41st IAS Annu. Meeting, Oct. 8-12, vol. 5, pp. 2608-2615.

[16] S. Muthu, F. J. Schuurmans, and M. D. Pashley, "Red, Green, and Blue LED based white light generation: Issues and control," in Proc. Conf. Record Ind. Appl. Conf., 2002, 37th IAS Annu. Meeting, Oct. 13-18, vol. 1, pp. 327-333.
[17] C. C. Chen, C. Y. Wu, and T. F. Wu, "Fast transition current-type burstmode dimming control for the LED back-light driving system of LCD TV," in Proc. 37th IEEE Power Electron. Spec. Conf., 2006, 18-22 Jun., pp. $1-7$.

[18] X. Xu and X. Wu, "High dimming ratio LED driver with fast transient boost converter," in Proc. IEEE Power Electron. Spec. Conf., 2008, 15-19 Jun., pp. 4192-4195.

[19] C. C. Chen, C. Y. Wu, Y. M. Chen, and T. F. Wu, "Sequential Color LED Backlight Driving System for LCD Panels," IEEE Trans. Power Electron., vol. 22, no. 3, pp. 919-925, May 2007.

[20] X. Qu, S. C. Wong, and Chi K. Tse, "Color control system for RGB LED light sources using junction temperature measurement," in Proc. 33rd Annu. Conf. IEEE Ind. Electron. Soc., 2007, Nov. 5-8, pp. 1363-1368.

[21] C-L. Chiu and K-H. Chen, "A high accuracy current-balanced contro technique for LED backlight," in Proc. IEEE Power Electron. Spec. Conf., 2008, 15-19 Jun., pp. 4202-4206.

[22] P. Narra and D. S. Zinger, "An effective LED dimming approach," in Proc. Conf. Record 2004 IEEE Ind. Appl. Conf., 2004, 39th IAS Annu. Meeting, Oct. 3-7, vol. 3, pp. 1671-1676.

[23] M. Dyble, N. Narendran, A. Bierman, and T. Klein, "Impact of dimming white LEDs: Chromaticity shifts due to different dimming methods," in Proc. 5th Int. Conf. Solid State Lighting, Proc. SPIE 5941, 2005, pp. 291299.

[24] Y. Gu, N. Narendran, T. Dong, and H. Wu, "Spectral and luminous efficacy change of high-power LEDs under different dimming methods," in Proc. 6th Int. Conf. Solid State Lighting, Proc. SPIE 6337, 2006, 63370J.

[25] L. Jayasinghe, L. Y. Gu, and N. Narendran, "Characterization of thermal resistance coefficient of high-power LEDs," in Proc. 6th Int. Conf. Solid State Lighting, Proc. SPIE 6337, 2006, 63370V.

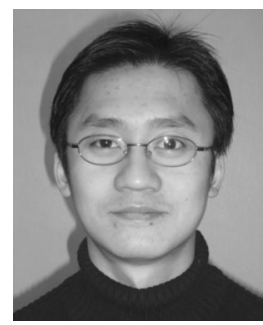

K. H. Loo (S'97-M'99) received the B.Eng. (Honors) degree in electronic engineering and the Ph.D. degree from the University of Sheffield, Sheffield, U.K., in 1999 and 2002, respectively.

From November 2002 to November 2004, he received the Postdoctoral Fellowship from the Japan Society for the Promotion of Science during which he carried out researches on the mercury-free fluorescent lamp diagnostics and modeling in Ehime University, Shikoku, Japan. From March 2005 to August 2006, he was an Electronic Engineer in a local lighting company in Hong Kong. In August 2006, he joined The Hong Kong Polytechnic University, Hong Kong, where he was an Instructor in the Faculty of Engineering. His current research interests include LED lighting and control circuitry designs, diagnostics, and modeling of fuel cells for portable applications.

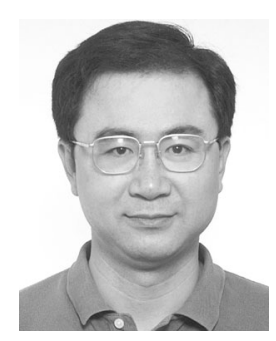

Y. M. Lai (M'92) received the B.Eng. degree in electrical engineering from the University of Western Australia, Perth, Australia, in 1983, the M.Eng.Sc. degree in electrical engineering from the University of Sydney, Sydney, Australia, in 1986, and the Ph.D. degree from Brunel University, London, U.K., in 1997.

He is currently an Assistant Professor at the Department of Electronic and Information Engineering, The Hong Kong Polytechnic University, Hong Kong, where he is engaged in research on computer-aided design of power electronics and nonlinear dynamics. 


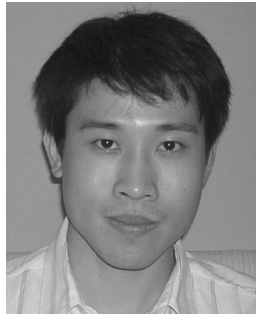

Siew-Chong Tan (S'00-M'06) received the B.Eng. (Honors) and M.Eng. degrees in electrical and computer engineering from the National University of Singapore, Singapore, in 2000 and 2002, respectively, and the Ph.D. degree from The Hong Kong Polytechnic University, Hong Kong, in 2005.

From October 2005 to February 2009, he was a Research Associate, Postdoctoral Fellow, and then as a Lecturer in the Department of Electronic and Information Engineering, The Hong Kong Polytechnic University, where he is currently an Assistant Professor. His research interests include nonlinear control of power converters, switched-capacitor converter circuits and control, and on developing powerelectronic-based circuits for light-emitting diodes and fuel cells.

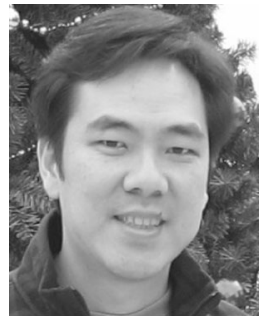

Chi K. Tse (M'90-SM'97-F'06) received the B.Eng. (First Class Honors) degree in electrical engineering and the Ph.D. degree from the University of Melbourne, Vic, Australia, in 1987 and 1991, respectively.

$\mathrm{He}$ is currently a Chair Professor and the Head of the Department of Electronic and Information Engineering, Hong Kong Polytechnic University, Hong Kong. He is the author of the Linear Circuit Analysis (London, U.K.: Addison-Wesley, 1998) and the Complex Behavior of Switching Power Converters (Boca Raton, FL: CRC Press, 2003), coauthor of the Chaos-Based Digital Communication Systems (Heidelberg, Germany: Springer-Verlag, 2003) and the Chaotic Signal Reconstruction With Applications to Chaos-Based Communications (Singapore: World Scientific, 2007). He is a coholder of a U.S. patent and two pending patents. His research interests include power electronics, complex networks, and nonlinear systems.

Prof. Tse has served as an Associate Editor for the IEEE TRANSACTIONS ON CIRCUITS AND SYSTEMS PART I-FUNDAMENTAL THEORY AND APPLICATIONS from 1999 to 2001, has been an Associate Editor for the IEEE TRANSACTIONS ON POWER ELECTRONICS since 1999, served as an IEEE Distinguished Lecturer in 2005. He also serves as the Editor-in-Chief of the IEEE Circuits and Systems Society Newsletter, Associate Editor for the IEEE TRANSACTIONS ON Circuits AND SYstems PART I-Regular PaPers, the IEEE Circuits AND SYSTEMS MAGAZINE, the International Journal of Systems Science, the International Journal of Circuit Theory and Applications, and a Guest Editor of a few other journals. He was awarded the L. R. East Prize by the Institution of Engineers, Australia, in 1987, the IEEE Transactions on Power Electronics Prize Paper Award in 2001, the International Journal of Circuit Theory and Applications Best Paper Award in 2003, and the Distinguished International Research Fellowship by the University of Calgary, Canada, in 2007. He received twice the President's Award for Achievement in Research, the Faculty's Best Researcher Award, the Research Grant Achievement Award, and a few other teaching awards. 